\title{
Surface Analysis of Fine Art Paintings: Studying Degradation Mechanisms with a Systematic Approach
}

\author{
Thomas P Beebe $\mathrm{Jr}^{1}$, Zachary Voras ${ }^{1}$, Kristin de Gheraldi ${ }^{3}$ and Jennifer Mass ${ }^{3}$
}

1. University of Delaware, Department of Chemistry \& Biochemistry, Newark DE, USA

2. University of Delaware and Winterthur Museum, Department of Art Conservation, Newark, DE, USA

3. Scientific Analysis of Fine Art LLC \& Rijksmuseum, Amsterdam, The Netherlands

A large body of work has been established in the field of pigment alteration covering several artistic eras [1-3]. While these studies are fundamental in understanding the physical and chemical properties pertaining to color as seen in paintings, they do not cover the important facet of molecular alteration due to degradation of the binding medium of these paintings. By using surface analytical techniques proven in the study of cultural heritage materials it is possible to elucidate the effects of degradation inherent to binding media and also add to the knowledge of pigment-binder interactions. For this study, chemical information gathered by both TOF-SIMS and XPS, a complete characterization of both short-range (XPS) and long-range (TOF-SIMS) molecular alteration related to egg tempera degradation is presented herein. By utilizing gas cluster ion beam (GCIB) technology with XPS it was possible to elucidate any changes in chemical oxidation and reduction as a function of depth for a given material and its exposure to its surroundings.

Thin films were prepared by spin casting freshly made egg tempera (1:1 w/w egg yolk:water) onto clean $1 \times 1$ inch silicon wafers. Samples were then placed as a set inside of a weathering chamber to simulate environmental degradation effects. Detailed in Figure 1, the chamber consists of an $\mathrm{N}_{2}$ purge, heat source, UV light source, and a relative humidity $(\mathrm{RH})$ source. The variables to start the degradation process were to be tested across two major sources: heat/humidity exposure and UV exposure. The three trials set forth in the experiment were to expose the egg tempera thin films to the following conditions: i) $60{ }^{\circ} \mathrm{C},>80 \%$ $\mathrm{RH}$, and a dark chamber; ii) $20^{\circ} \mathrm{C}, 0 \% \mathrm{RH}$, and UV exposure; and iii) $60^{\circ} \mathrm{C},>80 \% \mathrm{RH}$, and $\mathrm{UV}$ exposure.

XPS and GCIB depth profiling were conducted with a K-Alpha+ with MAGCIS (Thermo-Scientific, Inc.) located in the Surface Analysis Facility at the University of Delaware. Monochromated Al K $\alpha$ X-rays $(1486.6 \mathrm{eV})$ with a spot size of $100 \mu \mathrm{m}$ and pass energy of $20 \mathrm{eV}$ for all high resolution scans. GCIB profiling was done with a $4 \mathrm{kV} \mathrm{Ar} 2000$ cluster for $60 \mathrm{~s}$ each level of sputtering. All curve-fitting was done in CasaXPS using Shirley-type background correction. The sputter rate of the egg tempera was measured using atomic force microscopy and found to be $4.5 \times 10^{-3} \mathrm{~nm} / \mathrm{Ar}$ atom. TOF-SIMS was conducted with a TOF-SIMS IV (ION-TOF GmbH), upgraded to TOF-SIMS V capabilities, located in the Surface Analysis Facility at the University of Delaware. $25-\mathrm{kV} \mathrm{Bi}_{3}^{+}$primary ions were directed towards the sample surface in "high-current bunched mode" with secondary ion extraction with $\pm 2 \mathrm{kV}$ into the TOF mass analyzer and given a $10 \mathrm{kV}$ post-acceleration. All spectra were collected over an area of $250 \mu \mathrm{m}^{2}$ with primary ion beam dosage taken to the static SIMS limit of $1 \times 10^{12} \mathrm{ion} / \mathrm{cm}^{2}$. All data processing was completed in Matlab, using the correlated optimized warping (COW) package and the PLSToolbox. After COW alignment of the TOF-SIMS data to remove sample variance due to miscalibration, the data was preprocessed with mean centering and then analyzed with PCA.

XPS and GCIB depth profiling were used to identify any changes made to the oxidation state of the egg tempera film due to weathering, mainly pertaining to changes in triglycerides to free fatty acids. Shown 
here will be results from weathering conditions of $60{ }^{\circ} \mathrm{C},>80 \% \mathrm{RH}$, and UV exposure. TOF-SIMS was used to identify any changes in molecular composition changes of the fatty acid portion of the egg tempera films as previous research has indicated that alteration of the fatty acid composition is observed in historic egg tempera paint layers. Shown here will be TOF-SIMS negative mode results from masses $279-287$ $\mathrm{amu}$, which were used to monitor changes made to free/mono- stearic acid in relation to substitutions in the alkyl chain.

Figure 2 summarizes the top surface XPS results from the weathering conditions that simulate exposure to $60{ }^{\circ} \mathrm{C},>80 \%$ relative humidity, and UV exposure. Figure $2 \mathrm{a}$ is for all of the atomic species present, showing a small increase in oxygen relative abundance in comparison to a drop in both carbon and nitrogen relative abundances. As shown in Table $2 \mathrm{~b}$, the $\mathrm{C} 1 \mathrm{~s}$ region is a combination of both the above two weathering scenarios, with both an increase in the $\mathrm{CO} / \mathrm{CN}$ and $\mathrm{COOH}$ component, as well as removal of the $\mathrm{COO} / \mathrm{CON}$ component. Shown in Figure 2c are the fitted components of the top surface before depth profiling, and Figure $2 \mathrm{~d}$ are the results from the depth profile showing the change from degraded surface to unaltered bulk of the egg tempera film. The interface between degradation and unaltered bulk is estimated to be between levels 7 and 10 .

Figures 3 summarize the results of COW followed by PCA of only masses $279-287 \mathrm{amu}$ that are directly measuring stearic acid, either as a free fatty acid or as a fragment of a larger triglyceride. Figure 3 is the scores plot comparing PC 1 and PC 2 showing obvious grouping between the three weathering parameters and the control sample. Of note, the distribution between the UV exposure and the combination of $60^{\circ} \mathrm{C}$, $>80 \%$ relative humidity, and UV exposure have some overlap in their distribution in the scores plot, as well as the distinction of the $60{ }^{\circ} \mathrm{C},>80 \%$ relative humidity grouping. By looking at the loadings plots (not shown), the reason for variance can be explained as the addition of double bonds (unsaturation) in the alkyl chain in comparison to the saturated stearic acid molecular weight as identified with the arrow at $283.3 \mathrm{amu}$. As UV exposure is known to stimulate both hydrolysis and cross-linking of fatty acids, this molecular alteration is expected.

This study provided an initial look into the chemical pathways of surface molecular alteration in relation to degradation of fatty acid-containing binding media relating to environmental factors. The selective removal of intact free fatty acids as observed in various historic artworks has been linked to the hydrolysis of di- and triglyceride into free fatty acids which can diffuse through a paint layer towards the upper surface. By subjecting thin films of egg tempera to various parameters relating to ambient heat, relative humidity, and UV exposure, the identification of glyceride breakdown has been observed by XPS and TOF-SIMS analysis. For the first time in cultural heritage research, depth profile analysis using GCIB on the egg tempera films was used in conjunction with XPS to observe any changes in oxidation as a function of depth after weathering.

XPS analysis showed the oxidation of egg tempera into higher abundance of ester and amide functionality after head and humidity exposure, as well as a shift towards carboxylic functionality after UV exposure. This confirms the process of hydrolysis and breakdown of glyceride (and amino acid backbone) components of egg tempera. TOF-SIMS analysis provided a framework of molecular alteration pertaining to glyceride breakdown. By observing both molecular glyceride (in positive-ion mode) and stearic acid (in negative-ion mode) molecular fragments it was possible to observe trends in alteration due to environmental weathering. By using PCA the observation of greater unsaturation after weathering of stearic acid shows the great volatility of the free fatty acid components. 
References:

[1] L. Monico et al, Spectrochimica Acta Part A: Molecular and Biomolecular Spectroscopy 116 (2013), p. 270.

[2] F. Casadio, C. Daher, L. Bellot-Gurlet, Top Curr Chem (Z) 374 (2016), p.1.

[3] K. Janssens et al, Top Curr Chem (Z) 374 (2016), p. 1.

(1)

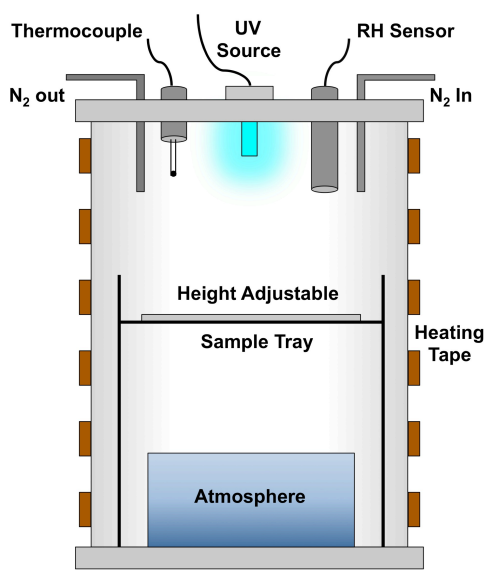

(2)

\begin{tabular}{|c|c|c|c|c|}
\hline \multicolumn{1}{|c}{ Day } & $\mathrm{C}$ & $\mathrm{O}$ & $\mathrm{N}$ & $\mathrm{P}$ \\
\hline 0 & $77.1 \pm 2.4$ & $19.9 \pm 1.8$ & $2.1 \pm 0.5$ & $1.0 \pm 0.2$ \\
\hline 1 & $75.8 \pm 0.1$ & $21.9 \pm 0.1$ & $0.9 \pm 0.1$ & $1.5 \pm 0.2$ \\
\hline 2 & $77.0 \pm 3.3$ & $21.6 \pm 2.3$ & $0.6 \pm 0.4$ & $0.8 \pm 0.6$ \\
\hline 3 & $80.1 \pm 1.7$ & $19.3 \pm 1.6$ & $0.2 \pm 0.05$ & $0.4 \pm 0.1$ \\
\hline 4 & $74.1 \pm 0.6$ & $24.1 \pm 0.8$ & $0.6 \pm 0.05$ & $1.2 \pm 0.3$ \\
\hline 5 & $73.1 \pm 0.5$ & $25.1 \pm 0.5$ & $0.8 \pm 0.1$ & $1.1 \pm 0.05$ \\
\hline
\end{tabular}

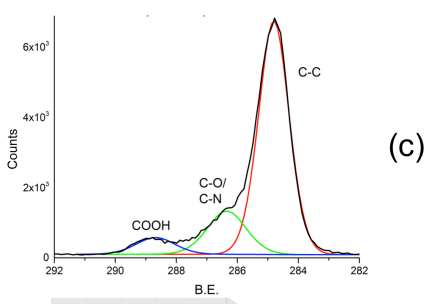

\begin{tabular}{|c|c|c|c|c|}
\hline \multicolumn{1}{c}{ Day } & \multicolumn{1}{c}{ COOH } & \multicolumn{1}{c}{ COO } & \multicolumn{1}{c|}{ CO/CN } & CC \\
\hline 0 & $2.6 \pm 0.6$ & $6.5 \pm 0.5$ & $18.3 \pm 1.8$ & $72.7 \pm 1.7$ \\
\hline 1 & $4.0 \pm 0.3$ & $3.8 \pm 0.4$ & $17.2 \pm 0.7$ & $75.0 \pm 0.5$ \\
\hline 2 & $5.2 \pm 0.7$ & $4.4 \pm 4.3$ & $20.1 \pm 9.3$ & $70.3 \pm 13.8$ \\
\hline 3 & $5.2 \pm 0.5$ & 0 & $14.8 \pm 2.5$ & $80.0 \pm 2.9$ \\
\hline 4 & $7.0 \pm 0.6$ & 0 & $20.2 \pm 1.6$ & $72.8 \pm 1.6$ \\
\hline 5 & $6.4 \pm 0.1$ & 0 & $20.1 \pm 1.4$ & $73.5 \pm 1.5$ \\
\hline
\end{tabular}

(c)

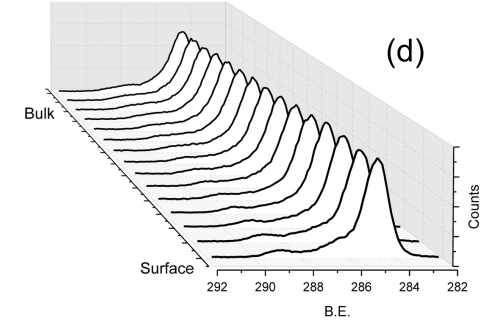

(3)

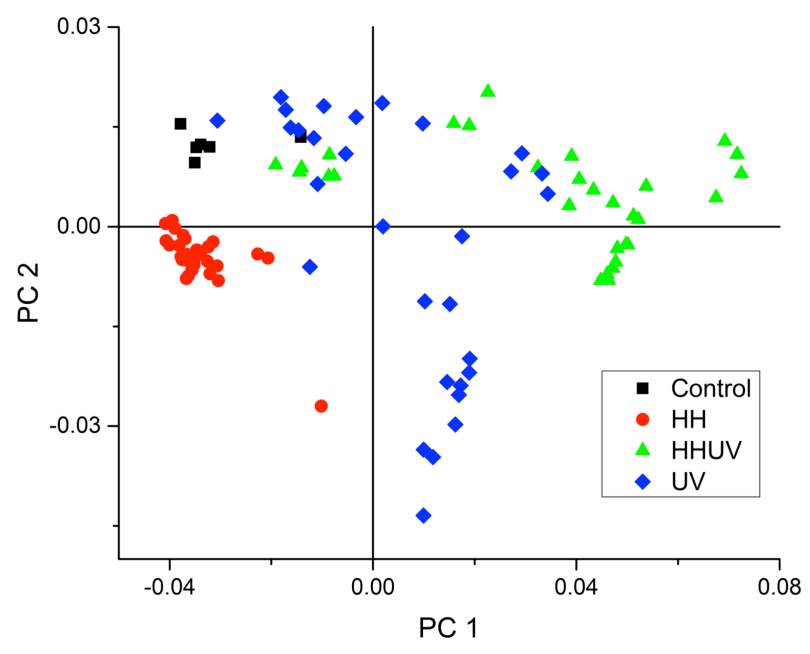

Figure 1. Weathering chamber used for the degradation of egg tempera thin films.

Figure 2. GCIB-XPS results of an egg tempera thin film weathered under conditions of $60{ }^{\circ} \mathrm{C},>80 \%$ $\mathrm{RH}$, and UV exposure.

Figure 3. Negative-mode TOF-SIMS results of all weathered egg tempera thin films. Only shown is the region of $279-287 \mathrm{amu}$. 
https://doi.org/10.1017/S1431927618005743 Published online by Cambridge University Press 